\title{
RESEARCH
}

\section{Nonsurgical management of partial adhesive small-bowel obstruction with oral therapy: a randomized controlled trial}

\author{
Shyr-Chyr Chen, Zui-Shen Yen, Chien-Chang Lee, Yueh-Ping Liu, Wen-Jone Chen, Hong-Shiee Lai, \\ Fang-Yue Lin, Wei-Jao Chen
}

\section{ABSTRACT}

Background: Patients with partial adhesive small-bowel obstruction are usually managed conservatively, receiving intravenous hydration and nothing by mouth. Previous studies have suggested that this approach is associated with longer hospital stays and an increased risk of delayed surgery. We conducted a randomized controlled trial to see if combining standard conservative treatment with oral administration of a laxative, a digestant and a defoaming agent would reduce the frequency of subsequent surgical intervention and reduce the length of hospital stay.

Methods: We identified 144 consecutive patients admitted between February 2000 and July 2001 with adhesive partial small-bowel obstruction and randomly assigned 128 who met the inclusion criteria to either the control group (intravenous hydration, nasogastric-tube decompression and nothing by mouth) or the intervention group (intravenous hydration, nasogastric-tube decompression and oral therapy with magnesium oxide, Lactobacillus acidophilus and simethicone). The primary outcome measures were the number of patients whose obstruction was successfully treated without surgery and the length of hospital stay. We also monitored rates of complications and recurring obstructions.

Results: Of the 128 patients, $\sigma_{3}$ were in the control group and $\sigma_{5}$ in the intervention group; the mean ages were 54.4 (standard deviation [SD] 15.9) years and 53.9 (SD 16.3) years respectively. Most of the patients were male. More patients in the intervention group than in the control group had successful treatment without surgery (59 [91\%] v. 48 [76\%], $p=$ 0.03 ; relative risk 1.19 , $95 \%$ confidence interval 1.03-1.40). The mean hospital stay was significantly longer among patients in the control group than among those in the intervention group (4.2 [SD 2.7] v. 1.0 [SD 0.7] days, $p<0.001$ ). The complication and recurrence rates did not differ significantly between the 2 groups.

Interpretation: Oral therapy with magnesium oxide, L. acidophilus and simethicone was effective in hastening the resolution of conservatively treated partial adhesive smallbowel obstruction and shortening the hospital stay.

CMAJ 2005;173(10):1165-9

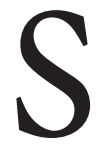

mall-bowel obstruction is usually caused by postoperative adhesions, which develop in about $95 \%$ of adult patients after abdominal surgery. ${ }^{1}$ Different factors, such as powder from surgical gloves and tissue retraction, have been reported to cause fibrous adhesions. ${ }^{2,3}$ Considerable controversy exists concerning the management of postoperative adhesive small-bowel obstruction..$^{1,4-8}$ Traditionally, conservative management involves intravenous hydration, decompression with a nasogastric tube and giving the patient nothing by mouth. ${ }^{9-12}$ Such treatment is reported to be successful in $73 \%-90 \%$ of cases. ${ }^{9,10,13}$ However, surgical treatment may be required in one-third of patients because of significant complications, such as strangulation, which can develop when surgery is delayed for more than 48 hours. ${ }^{11,14}$

Patients with partial adhesive small-bowel obstruction managed conservatively have a long hospital stay (usually between I and 3 weeks $8,15,16$ ), which is associated with increased hospital costs and an increased risk of delayed surgery. ${ }^{17}$ Speeding up the nonsurgical management may decrease the frequency of these problems. We performed a randomized controlled trial in which we compared the effects of standard conservative management with those of standard conservative management plus oral adminstration of a laxative, a digestant and a defoaming agent.

\section{Methods}

Our study was conducted in an emergency department of a tertiary care hospital in Taipei that serves about 100 ooo patients annually. The hospital's ethics committee for medical research approved the study protocol, and all of the patients provided their informed consent before inclusion in the study.

We considered all consecutive adult patients admitted between February 2000 and July 2001 with symptoms and signs suggestive of small-bowel obstruction to be eligible for inclusion in the study. The inclusion criteria were (a) a history of intra-abdominal surgery conducted more than 4 weeks before enrolment; (b) clinical symptoms and signs compatible with small-bowel obstruction, including abdominal pain, distension, nausea, vomiting and constipation; and (c) a plain abdominal radiograph taken with the patient upright that showed dilated loops of the small intestine, air fluid levels and gas in the colon, indicating a partial small-bowel obstruction. ${ }^{13}$ 
We did not include patients with a complete small-bowel obstruction (no clear evidence of air in the large bowel on abdominal radiographs). Specifically, we excluded patients if they had one or more signs suggestive of intestinal strangulation or obstruction present at admission, including fever, intractable pain, leukocytosis or signs of peritonitis. In addition, we excluded patients with a partial small-bowel obstruction if the cause was something other than adhesions (e.g., inflammatory bowel disease, external hernia, malignant disease or prior abdominal irradiation), the abdominal radiograph showed no gas in the colon, the patient was less than I5 years old or the obstruction developed within the first 4 weeks after abdominal surgery.

Upon arrival to the emergency department, each patient was evaluated by a member of the emergency department staff. After taking a detailed history and performing a physical examination, the staff member drew a venous blood sample for complete blood cell count and took a plain abdominal radiograph with the patient in an upright position. If the attending emergency physician confirmed that it was a case of partial adhesive small-bowel obstruction, the patient was considered for inclusion in the study. Eligible patients were randomly assigned to one of 2 groups by the attending emergency physician at the time of hospital admission. The randomization was performed by drawing a sealed, opaque envelope from a box containing an equal number of cards labelled either $\mathrm{N}$ (nothing by mouth) or OM (oral medications). The

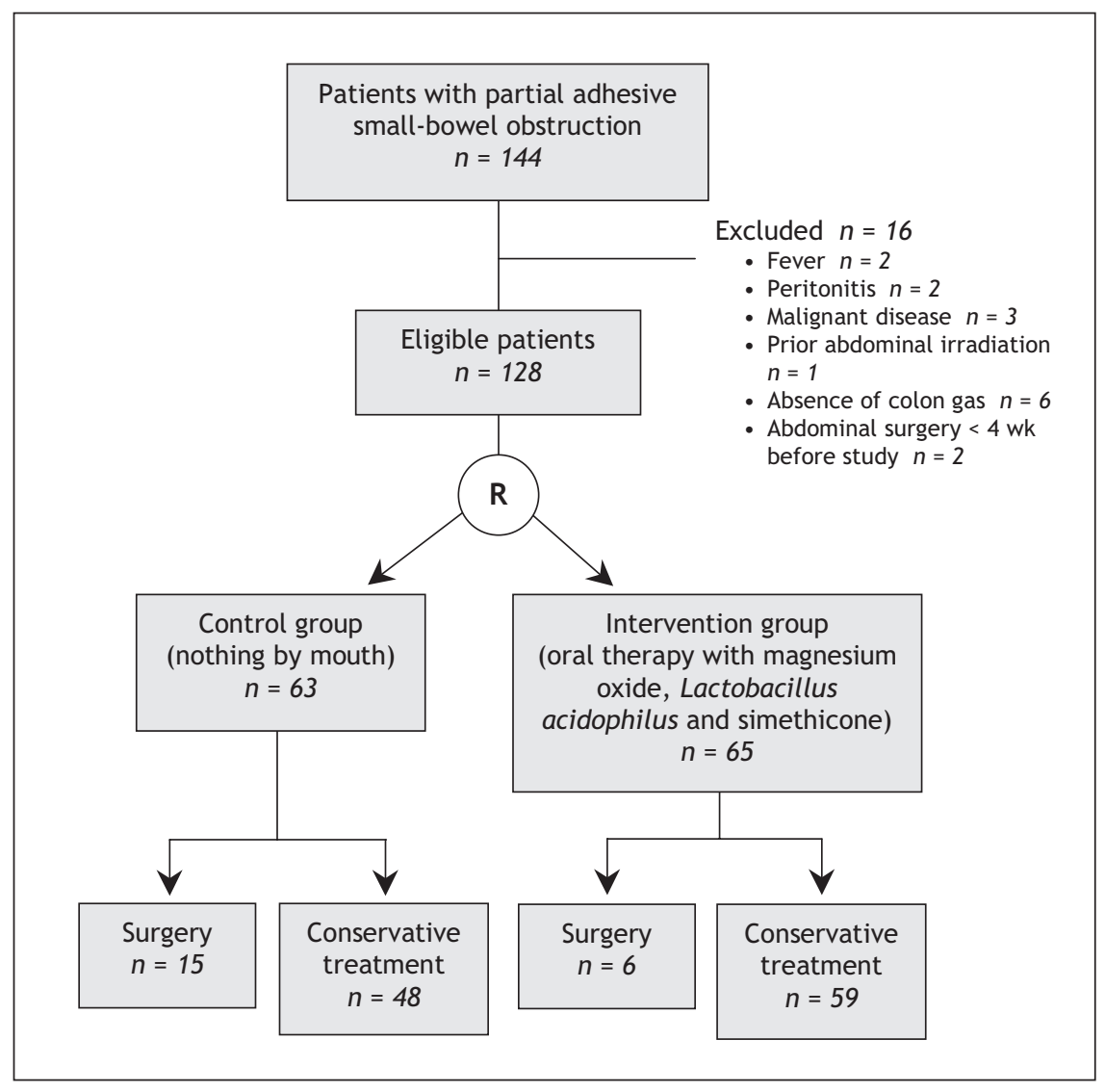

Fig. 1: Flow of patients through the trial. attending surgeon was blind to the patient allocation.

All patients were managed by means of intravenous hydration with Ringer's lactate solution and nasogastric-tube decompression. Patients in the control group received nothing by mouth, and those in the intervention group were given water and oral therapy with a laxative (two 250-mg tablets of magnesium oxide), a digestant (one 0.3-g tablet of Lactobacillus acidophilus) and a defoaming agent (one 40mg tablet of simethicone) 3 times daily. The nasogastric tube was clamped for one hour after the oral administration of the medications, to prevent reflux of the medications through the tube. The clamped portion of the tube was covered with a blanket to keep the attending surgeon blinded to the patient allocation.

The attending surgeon regularly assessed the patient's condition during the hospital stay to ensure that the nonsurgical treatment was going well and that no symptoms or signs developed that would suggest the need for surgery. Surgical intervention in both groups was determined by the attending surgeon based on the presence of one or more toxic signs (e.g., fever, leukocytosis, intractable pain and peritonitis) or if the obstruction did not resolve spontaneously after 5 days.

Patients in both groups underwent plain abdominal radiography daily. The nasogastric tube was removed when oral intake of food was started. Oral intake was initiated with a liquid diet followed by a soft diet after resolution of the adhesive small-bowel obstruction was confirmed radiographically, the abdominal pain subsided or the patient passed stools or flatus.

Patients were discharged from the hospital when the following criteria were met: (a) the abdominal pain subsided and a solid diet was tolerated, and (b) a plain abdominal radiograph showed the absence of gas in the small bowel. The oral therapy was stopped at discharge in the intervention group. After discharge, patients were regularly followed up at an outpatient clinic for 6 months.

We recorded the patient's age, sex and type of abdominal surgery as well as his or her symptoms and signs on admission. The primary outcome measures were the number of patients whose obstruction was successfully treated without surgery and the length of hospital stay, which included the time from surgery to discharge for patients who subsequently required surgical treatment. We also recorded complications and recurrence of symptoms during the 6-month follow-up period.

Using a significance level of 0.05 and a power of $80 \%$, we determined that $50 \mathrm{pa}-$ tients were needed in each group to detect a difference in the reduction of hospital stay between the 2 groups of at least 2 days (since, in our experience, a typical length of hospital stay of patients with partial ad- 
hesive small-bowel obstruction is usually $3-7$ days). We used the $\chi^{2}$ test to compare differences in proportions and the Student $t$ test to compare continuous variables.

\section{Results}

During the study period I44 consecutive adult patients with partial adhesive small-bowel obstruction were admitted to the emergency department. Sixteen met the exclusion criteria, and the remaining 128 patients agreed to participate in the study (Fig. I). Patients in the 2 groups had similar characteristics, including age, sex and clinical presentation (Table I). Most of the patients had abdominal pain, distension and constipation. Vomiting was noted in less than $50 \%$. All of the pa-

Table 1: Demographic characteristics of 128 patients with partial adhesive small-bowel obstruction receiving nothing by mouth (control) or oral therapy (intervention)*

\begin{tabular}{|c|c|c|}
\hline \multirow[b]{2}{*}{ Characteristic } & \multicolumn{2}{|c|}{ Group; no. of patients $†$} \\
\hline & $\begin{array}{l}\text { Control } \\
n=63\end{array}$ & $\begin{array}{c}\text { Intervention } \\
\quad n=65\end{array}$ \\
\hline \multicolumn{3}{|l|}{ Sex } \\
\hline Male & 34 & 36 \\
\hline Female & 29 & 29 \\
\hline \multicolumn{3}{|l|}{ Age, yr } \\
\hline Mean (SD) & $54.4(15.9)$ & $53.9(16.3)$ \\
\hline Median (range) & $56(20-81)$ & $55(18-83)$ \\
\hline \multicolumn{3}{|l|}{ Symptom } \\
\hline Abdominal pain & 62 & 63 \\
\hline Distension & 53 & 54 \\
\hline Constipation & 49 & 50 \\
\hline Vomiting & 31 & 30 \\
\hline Previous surgery & 67 & 68 \\
\hline \multicolumn{3}{|l|}{ Upper abdomen } \\
\hline Subtotal gastrectomy & 11 & 11 \\
\hline $\begin{array}{l}\text { Truncal vagotomy and } \\
\text { pyloroplasty }\end{array}$ & 8 & 10 \\
\hline Cholecystectomy & 5 & 4 \\
\hline Hepatectomy & 3 & 2 \\
\hline Splenectomy & 1 & 1 \\
\hline Other & 3 & 2 \\
\hline \multicolumn{3}{|l|}{ Lower abdomen } \\
\hline Appendectomy & 13 & 14 \\
\hline Colectomy & 11 & 10 \\
\hline Abdominal total hysterectomy & 5 & 7 \\
\hline Salpingectomy & 2 & 3 \\
\hline $\begin{array}{l}\text { Abdominal total hysterectomy } \\
\text { with salpingectomy }\end{array}$ & 3 & 2 \\
\hline Cesarean section & 1 & 1 \\
\hline Other & 1 & 1 \\
\hline
\end{tabular}

Note: SD = standard deviation.

*Oral therapy comprised magnesium oxide, Lactobacillus acidophilus and simethicone.

†Unless stated otherwise. tients had undergone previous abdominal surgery (Table I), 7 of whom had undergone more than one operation.

Table 2 shows the outcomes for the 2 groups. The number of patients whose obstruction was successfully treated without surgery was significantly higher in the intervention group than in the control group (59 [91\%] v. 48 [76\%]; relative risk I.I9, $95 \%$ confidence interval [CI] I.03-I.40). The mean length of hospital stay was significantly longer in the control group than in the intervention group ( $4.2 \mathrm{v}$. I.o days, $p<0.00 \mathrm{I}$ ). Of the patients in the intervention group, 28 had spontaneous passage of stool and $4 \mathrm{I}$ had improved abdominal distension.

Two patients in the control group had complications (nostril erosion and pneumonia) but did not require surgical treatment. In the intervention group, 2 patients had complications (urinary tract infection and recurrent obstruction), and another patient who subsequently required surgical treatment had aspiration pneumonia as a postoperative complication. None of the complications in the intervention group was related to the investigated treatment with magnesium oxide, $L$. acidophilus and simethicone.

During the 6-month follow-up, partial adhesive smallbowel obstruction recurred in 4 patients in the control group 3 had been managed conservatively and I had required surgical treatment) and 3 patients in the intervention group ( 2 had been managed conservatively and I had required surgical treatment) (Table 2). None of these patients required surgery for the recurrence. Three patients were lost during the 6-month follow-up.

Of the 2I patients who required surgical treatment, 6 had segmental bowel resection for bowel ischemia and bowel injury, and I5 required adhesiolysis (Table 3 ). Surgery was performed on the third day after admission for 3 patients, the fourth day for 6 patients and the fifth day for 12 patients. The most common reason for surgery was peritonitis $(n=7)$; other reasons included fever, failure of treatment (i.e, no improvement after 5 days), leukocytosis and intractable pain (Table 3). The postoperative courses were unremarkable, and no postoperative deaths occurred.

\section{Interpretation}

We found that adding oral therapy with magnesium oxide, $L$. acidophilus and simethicone to the standard nonsurgical treatment of partial adhesive small-bowel obstruction re-

Table 2: Frequency of surgery, length of hospital stay, occurrence of complications and recurrence of small-bowel obstruction

\begin{tabular}{lccc}
\hline & \multicolumn{2}{c}{ Group; no. of patients* } & \\
\cline { 2 - 3 } & $\begin{array}{c}\text { Control } \\
n=63\end{array}$ & $\begin{array}{c}\text { Intervention } \\
n=65\end{array}$ & $p$ value \\
\hline Outcome & 15 & 6 & 0.031 \\
\hline $\begin{array}{l}\text { Surgery needed } \\
\begin{array}{l}\text { Length of hospital stay, } \\
\text { d, mean (SD) }\end{array}\end{array}$ & $4.2(2.7)$ & $1.0(0.7)$ & $<0.001$ \\
$\begin{array}{l}\text { Complications } \\
\begin{array}{l}\text { Recurrence of small-bowel } \\
\text { obstruction }\end{array}\end{array}$ & 2 & 3 & 1 \\
\hline
\end{tabular}

*Unless stated otherwise. 
Table 3: Characteristics of patients with partial adhesive smallbowel obstruction who underwent surgery

\begin{tabular}{lcc}
\hline & \multicolumn{2}{c}{ Group; no. of patients } \\
\cline { 2 - 3 } & $\begin{array}{c}\text { Control } \\
n=15\end{array}$ & $\begin{array}{c}\text { Intervention } \\
n=6\end{array}$ \\
\hline Characteristic & & \\
\hline Surgical method & 4 & 2 \\
\hline Bowel resection & 11 & 4 \\
\hline Adhesiolysis & & \\
\hline Day of surgery after admission & 2 & 1 \\
\hline Third & 5 & 1 \\
\hline Fourth & 8 & 4 \\
\hline Fifth & & \\
\hline Surgical indication & 4 & 3 \\
\hline Peritonitis & 4 & 1 \\
\hline Fever & 3 & 1 \\
\hline Failure of treatment & 2 & 1 \\
\hline Leukocytosis & 2 & 0 \\
\hline Intractable pain & & \\
\hline
\end{tabular}

sulted in a marked reduction in the need for surgical intervention and the length of hospital stay compared with the standard nonsurgical treatment alone. The number needed to treat to avoid one surgical treatment was 7 ( $95 \%$ CI 3.7-52).

Previous studies of the management of small-bowel obstruction focused on improvements in patient assessment to better identify those who would need surgical treatment. In our earlier nonrandomized studies, ${ }^{18,19}$ we found that water-soluble contrast medium can be used to determine whether the obstruction is partial or complete and whether nonsurgical treatment has been successful. Biondo and colleagues ${ }^{20}$ conducted a randomized controlled trial of the radiocontrast material Gastrografin and showed that passage of the contrast material beyond the obstruction reliably predicted subsequent recovery without surgery. Our study contributes to this literature by showing in a randomized controlled trial that the oral administration of a laxative, a digestant and a defoaming agent leads to improved outcomes and that the number needed to treat is very low (7 patients).

The oral therapy we used may have been effective because it included magnesium oxide, which stimulates bowel movement and subsequently causes the bowels to empty. Laxatives may have side effects such as increased pain and diarrhea, particularly in patients with bowel obstruction; however, we did not find this to be the case in our study, although it should be noted that we did not use a prokinetic agent, which might have otherwise increased peristalsis. We did use a defoaming agent, which alters surface tension of gas bubbles and causes them to coalesce, thus accelerating the passage of gas through the intestinal tract and consequently reducing gaseous symptoms. ${ }^{21-23}$

Because small-bowel obstruction is characterized by impeded bowel peristalsis followed by stasis of gastric and intestinal juices and food, we used $L$. acidophilus to help with the digestion of food debris that was probably not completely digested in the bowel lumen. The effect of the obstruction on the bowel is simi- lar to that of constipation and abdominal distension, so we chose dosages for the 3 medications that were the same as those used to treat constipation and abdominal distension. Spontaneous passage of stool and improved abdominal distension in our intervention group may imply that the medications helped the recovery of bowel peristalsis and passage of gas.

Delayed surgery in patients with partial adhesive smallbowel obstruction managed conservatively may be a source of concern because of the increased risk of complications. However, neither the morbidity nor the mortality rate was increased in our intervention group, which indicates the safety of this therapy.

Our study has several limitations. First, although it was prospective and randomized, it was not double-blinded, which leaves room for potential investigator bias in patient management. Second, the cases of partial adhesive smallbowel obstruction were suspected on the basis of the patient's clinical presentation and medical history and confirmed with the use of plain radiography, but they were not definitively proven with contrast radiography. Therefore, some of the cases successfully treated with the oral therapy may not have been caused by adhesion. Third, although the patients were randomly assigned to the groups and the attending surgeon was blinded to their allocation, the attending surgeon may have seen the clamp on the nasogastric tube when the oral therapy was being administered and learned to which group a patient was assigned. Fourth, because this study was conducted at only one institution, the generalizability of our findings to other institutions remains unknown.

Compared with the traditional conservative management of partial adhesive small-bowel obstructions, in which patients are given nothing by mouth, the addition of oral therapy with a laxative, a digestant and a defoaming agent leads to a quicker resolution and a shorter hospital stay. Further trials with larger patient samples are needed to verify the value of oral therapy for partial adhesive small-bowel obstructions observed in this study.

\section{Editor's take}

- Partial adhesive small-bowel obstruction is usually managed conservatively by giving patients nothing by mouth and waiting to see if the obstruction resolves spontaneously.

- In this study consecutive patients were randomly assigned to usual management or management with oral administration of a laxative, a digestant and a defoaming agent.

- Patients given the oral therapy were more likely than the control subjects to have spontaneous resolution without the need for surgery (relative risk 1.19, 95\% confidence interval 1.03-1.40) and to have shorter hospital stays.

- There were no serious adverse events from the oral therapy.

Clinical implications: Oral therapy appears to be helpful in the management of patients with partial adhesive small-bowel obstruction. 
This article has been peer reviewed.

From the Departments of Emergency Medicine (Shyr-Chyr Chen, Yen, Lee, Liu, Wen-Jone Chen) and Surgery (Lai, Lin, Wei-Jao Chen), National Taiwan University Hospital and National Taiwan University College of Medicine, Taipei, Taiwan

Competing interests: None declared.

Contributors: Shyr-Chyr Chen was the principal author and was responsible for the study design, managing and monitoring the study and contributing to the analysis and interpretation of the data. Zui-Shen Yen, Chien-Chang Lee, Yueh-Ping Liu and Wen-Jone Chen helped with the study design and the monitoring of the study and contributed to the analysis and interpretation of the data. Hong-Shiee Lai, Fang-Yue Lin and Wei-Jao Chen helped refine the study design and contributed to the analysis and interpretation of the data. All of the authors participated in revising the manuscript and approved the final submission.

Acknowledgement: We thank Professor Yeu-Sheng Hsieh, Department of Agricultural Extension, National Taiwan University, for his help with the statistical analysis.

\section{REFERENCES}

I. Menzies D, Ellis H. Intestinal obstruction from adhesions: How big is the problem? Ann R Coll Surg Engl I990;72:60-3.

2. Ellis H. Adhesions: an introduction. In: Adhesions: the problems. Ellis H, Lennox M, editors. London (UK): Westminster Hospital Medical School; I983. p. I-5.

3. Holmdahl L, Risberg B. Adhesions: prevention and complications in general surgery. EurJ Surg 1997;163:169-74.

4. Nieuwenhuijzen M, Reijnen MMPJ, Kuijpers JH, et al. Small bowel obstruction after total or subtotal colectomy: a Io-year retrospective review. Br J Surg I998;85:1242-5.

5. Richards WO, William LF Jr. Obstruction of the large and small intestine. Surg Clin North Am I988;68:355-76.

6. Feigin E, Seror D, Szold A, et al. Water-soluble contrast material has no therapeutic effect on postoperative small-bowel obstruction: results of a prospective, randomized clinical trial. Am J Surg I996;171:227-9.

7. Ellis $\mathrm{H}$. The clinical significance of adhesions: focus on intestinal obstruction. Eur J Surg Suppl I997;557:5-9.

8. Miller G, Shrier BI, Gordon PH. Natural history of patients with adhesive small bowel obstruction. BrJ Surg 2000;87:1240-7.

9. Seror D, Feigin E, Szold A, et al. How conservatively can postoperative small bowe obstruction be treated? Am J Surg 1993;165:121-6

Io. Assalia A, Schein M, Kopelman D, et al. Therapeutic effect of oral Gastrografin in adhesive, partial small-bowel obstruction: a prospective randomized trial. Surgery I994;II5:433-7.

II. Sosa J, Gardner B. Management of patients diagnosed as acute intestinal obstruction secondary to adhesions. Am Surg 1993;59:125-8.

I2. Cox MR, Gunn IF, Eastman MC, et al. The safety and duration of non-operative treatment for adhesive small bowel obstruction. Aust N Z J Surg 1993;63:367-7I.

I3. Brolin RE. Partial small bowel obstruction. Surgery 1984;95:145-9.

14. Shih SC, Jeng KS, Lin SC, et al. Adhesive small bowel obstruction: How long can patients tolerate conservative treatment? World J Gastroenterol 2003;9:603-5.

15. Brolin RE, Krasna MJ, Mast BA. Use of tubes and radiographs in the management of small bowel obstruction. Ann Surg 1987;206:126-33.

I6. Asbun HJ, Pempinello C, Halasz NA. Small bowel obstruction and its management. Int Surg 1989;74:23-7.

17. Jeekel H. Cost implications of adhesions as highlighted in a Europen study. Eur J Surg Suppl 1997;(579):43-5.

18. Chen SC, Lin FY, Lee PH, et al. Water-soluble contrast study predicts the need for early surgery in adhesive small bowel obstruction. Br J Surg I998;85:1692-4.

19. Chen SC, Chang KJ, Lee PH, et al. Oral Urografin in postoperative small bowel obstruction. World J Surg I999;23:105I-4.

20. Biondo S, Pares D, Mora L, et al. Randomized clinical study of Gastrografin administration in patients with adhesive small bowel obstruction. BrJ Surg 2003;90:542-6.

2I. Danhof IE, Stavola JJ. Accelerated transit of intestinal gas with simethicone. Obstet Gynecol I974;44(I):I48-54.

22. Danielesson B, Hwang CP. Treatment of infantile colic with surface active substance (simethicone). Acta Pediatr Scand ig85;74:446-50.

23. Metcalf TJ, Irons TG, Sher LD, et al. Simethicone in the treatment of infant colic: randomized placebo-controlled, multicenter trial. Pediatrics 1994;94:29-34.

Correspondence to: Dr. Wei-Jao Chen, Department of Surgery, National Taiwan University Hospital and National Taiwan University College of Medicine, 7 Chung-Shan South Rd., Taipei Ioor6, Taiwan; fax +886-2-23223150; scc@ha.mc.ntu.edu.tw 